\title{
Importancia de las prácticas pre profesionales para los estudiantes de educación superior en la Universidad de Guayaquil
}

\section{Importance of pre-professional practices for students of higher education at the University of Guayaquil}

\author{
Steve Freddy Guarnizo Crespo \\ Universidad de Guayaquil, Ecuador
}

Autor para correspondencia: steve.guarnizoc@ug.edu.ec

Fecha de recepción: 7 de abril de 2018 - Fecha de aceptación: 15 de agosto de 2018

Resumen: EL presente documento busca determinar la importancia de las practicas pre profesionales en los estudiantes de educación superior en la universidad de Guayaquil, relacionadas con su preparación para la inserción laboral, enfocando diversas posturas, conceptualizaciones, opiniones, criterios, en lo que se refiere esta actividad complementaria de la formación académica en los estudiantes universitarios, así como también busca analizar la interacción de los actores que intervienen en este proceso de formación estudiantil. El alcance de esta investigación engloba a los principales actores de este proceso académico, los cuales involucran a las reglamentaciones impuestas por el estado hacia las instituciones de educación superior, y a todos los actores de la universidad como son personal administrativo, docente y alumnos los cuales de manera interactiva desarrollan la formación práctica que realiza el estudiante en su trayectoria académica. El presente documento tiene como finalidad proponer la concientización, de los actores involucrados en este proceso de prácticas pre profesionales, de manera que se comprenda el beneficio de una buena ejecución del desarrollo de la práctica en beneficio del alumno para enfrentarse a las diversas expectativas que se ofrecen en el mercado laboral Palabras Claves: practicas pre profesionales; formación académica; formación práctica inserción laboral; mercado laboral; procesos académicos

\begin{abstract}
This document seeks to determine the importance of pre-professional practices in higher education students at the University of Guayaquil, related to their preparation for employment, focusing on different positions, conceptualizations, opinions, criteria, in what concerns This activity complements the academic training of university students, as well as seeks to analyze the interaction of the actors involved in this process of student education. The scope of this research encompasses the main actors of this academic process, which involve the regulations imposed by the state towards higher education institutions, and all the actors of the university, such as administrative staff, teachers and students. Interactive way they develop the practical formation that the student makes in his academic trajectory. The purpose of this document is to propose the awareness of the actors involved in this process of pre-professional practices, so as to understand the benefit of a good execution of the development of the practice for the benefit of the student to face the different expectations that are offer in the labor market
\end{abstract}

Key words: pre-professional practices; academic training; practical training; job placement; labor market; academic processes 


\section{Introducción}

En el mundo laboral del presente es necesario tener un buen rendimiento laboral, debido a que las empresas exigen profesionales que sean eficientes en desarrollar los distintos procesos necesarios para alcanzar los objetivos de la compañía. Para tener un buen rendimiento laboral existen varios determinantes, en primer lugar, se encuentra la formación académica, pues es de suma importancia la adquisición de conocimientos obtenidos en la universidad, también otro determinante es el desarrollo de competencias tanto técnicas como transversales, y éstas no se desarrollan solamente en la universidad, sino que también son potenciadas y/o adquiridas, en el contexto de las prácticas (Ferreyra, 2007).

Los autores Gault, Redington, y Schlager (2000) describen que un típico programa de prácticas se caracteriza por tres criterios: (1) un número determinado de horas trabajadas, (2) el trabajo puede ser pagado o no remunerado, (3) la supervisión es proporcionada por un coordinador de la facultad u otro representante de la universidad y una contraparte corporativa. La compensación es por lo general requerida para las empresas, pero puede ser opcional para algunos programas (DiLorenzo-Aiss, 1996)

Las practicas pre profesionales como bien dice su nombre es una alternativa de oportunidad laboral que recibiría un estudiante ante uno de los principales fines de formación en una institución de educación superior.

El estudiante de una IES, busca culminar su carrera para enfrentar un reto en el mercado laboral, para mejorar una posición en el trabajo dentro de las diversas jerarquías que se dan en una organización, sin embargo a medida que pasa el tiempo y el estudiante avanza en sus diferentes niveles de formación académica, surge una pregunta, ¿Con lo que he aprendido en el salón de clases, estoy preparado para ocupar un cargo en una empresa?, ¿con el título que voy a obtener, realmente puedo aspirar a una posición de alta jerarquía en una organización?.

En el Ecuador en los diversos anuncios publicitarios para búsqueda de futuros candidatos, en el mejor de los casos piden experiencia laboral, lo cual limita a la oportunidad de trabajo de estos posibles candidatos, en concordancia con el párrafo anterior, las incógnitas se responden solas ante esa situación, "no estoy preparado".

Se puede entonces entender que gran cantidad de los estudiantes de la IES, son personas que en unos casos tienen poca o ninguna experiencia laboral, por lo que las practicas pre profesionales sería un aporte medular para la inserción de los estudiantes al mercado laboral, teniendo como resultado una consolidación entre la academia y la práctica laboral real.

Muchas empresas por otro lado, no comprenden este sentido de la práctica pre profesional, es importante un acercamiento por parte de las IES, para socializar el espíritu del desarrollo de la práctica pre profesional, darles a conocer lo que realmente se busca con esta actividad académica, el desconocimiento de esta finalidad por parte de los empresarios, se ve muchas veces reflejado en las actividades o tareas que se le encomienda al estudiante al realizar o ejecutar la práctica, tareas de poca importancia, que no ayudan a desarrollar lo aprendido en su formación académica. 


\section{Antecedentes de la práctica profesional en la educación superior}

La formación práctica no solo dice relación con la preparación más integral de profesionales y técnicos, sino que también con una concepción teórica de la educación y del currículo. En esta postura los nuevos saberes se construyen mediante una relación hermenéutica entre un objeto de aprendizaje de carácter interno (conformada como una imagen ideal en el intelecto del individuo) y la realidad externa y objetiva. La interacción entre ambos genera un nuevo conocimiento que a su vez modifica tanto la realidad interna como la externa (Rodríguez \& González, 1995).

En esta concepción educativa se valora de manera preponderante la experiencia, el aprendizaje inductivo basado en la sistematización de la práctica y el constructivismo social en el cual tanto docentes como discentes aportan a la elaboración de un nuevo saber compartido.

Desde la perspectiva señalada, todo acto pedagógico se constituye en un proceso dialéctico de acción y reflexión, en una actividad integrativa entre teoría y práctica. El concepto de formación práctica con frecuencia se entiende como el periodo de prácticas formativas incluido en las carreras universitarias hacia el final del plan de estudios, entre ellas, las prácticas de enseñanza (Derrick \& Dicks, 2005) y prácticas en empresas (Collinson, y otros, 2009). Sin embargo, como se ha señalado, la formación práctica no es solo una actividad curricular que se dé al final de la carrera, o como una asignatura complementaria del plan de estudio, sino que forma parte del quehacer cotidiano, que se desarrolla en forma permanente en cada uno de los procesos de aprendizaje que se dan en un programa de formación y dentro de la actividad docente universitaria en general.

Si bien el enfoque educativo antes señalado puede darse en cualquier actividad formativa, no cabe duda de que existen carreras que se prestan más para este tipo de actividad. Se trata de carreras más orientadas al desempeño y la aplicación de conocimientos en las cuales el currículo está más centrado en la formación práctica, como es el caso de las profesiones de la salud, pedagogía e ingeniería, entre otras, que además realizan actividades de formación práctica a partir de etapas tempranas del currículo (Zabalza Beraza, M.A., 2011).

En general, en las distintas carreras la formación práctica varía en su profundidad, amplitud y realismo a medida que se avanza en los años de un plan de estudios, contando en algunos casos con estrategias de progresión de práctica claramente definidos. Existen, por un lado, ejercicios en el aula, talleres, laboratorios y visitas a terreno, por nombrar algunas, y por otro, prácticas profesionales, internados, prácticas de verano, etc. Las líneas curriculares con un componente de formación práctica y sus objetivos debieran operar de manera integrada en función de las orientaciones del perfil de egreso de la carrera, lo cual no siempre ocurre (Zabalza Beraza, 2006).

Las necesidades de nuestra sociedad son cada vez más específicas y concretas, lo que significa que la formación profesional debe estar en caminada en el mismo sentido. El vínculo universidad-comunidad se establece en el compromiso social de la primera en satisfacer la demanda de personas preparadas y capaces de enfrentar los problemas sociales que afectan a la 
segunda. En este sentido las universidades han cambiado su mirada hacia la profesionalización de sus carreras y han establecido la importancia de formar personas no sólo con el conocimiento adecuado para entender la realidad social sino también, capaces de enfrentarla y modificarla, para ello la importancia de formar a partir de la práctica (Yáñez, Soto, Domínguez, \& Hacegaba, 2014).

Estas conceptualizaciones dan a interpretar muchas aplicaciones, las cuales en diversas universidades se desarrollan según las interpretaciones de posibles conocedores de esta actividad, perdiendo de esta manera la verdadera intención de la creación de esta herramienta académica en el estudiante de las IES.

En diversas ocasiones se ha visto que el estudiante se encuentra realizando actividades de ayudantía con el docente, es decir está realizando actividades de prácticas dentro de la IES, como en la conceptualización del RRA (reglamento de régimen académico), menciona que lo puede realizar en el entorno institucional, muchas instituciones educativas lo tomaron literalmente, causando un grave perjuicio al espíritu de esta actividad.

En resumen, se puede inferir según las conceptualizaciones revisadas, que se busca extercionalizar el conocimiento adquirido en las aulas en el ambiente laboral, por lo que la práctica pre profesional no se debería ejercer en la IES, puesto que el estudiante no estaría ejerciendo su conocimiento en un ambiente real, que es la finalidad de esta herramienta educativa.

\section{Características de los actores de las prácticas pre profesionales: la universidad, los alumnos, las empresas}

Con la incorporación de las TIC's a la educación, y las posibilidades que ofrecen en la comunicación, se produce un cambio en el modelo pedagógico que comporta que los estudiantes pasen de ser meros receptores a convertirse también ellos en emisores $\mathrm{y}$, por tanto, forman parte activa del proceso de enseñanza y aprendizaje. De hecho, se les considera como los verdaderos protagonistas del acto educativo, al servicio de los cuales el resto de variables determinantes en los procesos didácticos quedan pendientes: docentes, materiales de aprendizaje y medios tecnológicos.

La formación en el puesto de trabajo necesaria para la especialización, la educación continua imprescindible para la adaptación a los cambios en el entorno laboral, la introducción y el uso de las TIC'S, y el resto de fenómenos derivados de los cambios sociales, repercuten directamente en el papel que los estudiantes ejercen en los procesos educativos. En el marco actual los alumnos dejan de ser personajes que reciben una formación puntual que les capacita, para convertirse en elementos activos que puedan responder a las demandas de la sociedad en cuanto a actualización constante de los conocimientos, procedimientos, actitudes y habilidades, el conjunto de lo cual ha constituido el concepto de competencia.

En este contexto el estudiante se convierte en el responsable de su propio proceso formativo, y como tal actúa para dar respuesta a sus necesidades personales, caracterizadas por aspectos individuales diferenciales respecto el grupo de iguales. A su vez, el alumno marca el 
ritmo de su propio aprendizaje y organiza su tiempo dedicando una parte de él a la formación, al no depender de límites marcados por agendas o calendarios fijados por las instituciones competentes ni del espacio físico.

La superación de las barreras espacio-temporales intrínsecas a la educación presencial convencional, permite la configuración de nuevos colectivos de estudiantes con características diferenciales al poder compatibilizar la formación con el trabajo, la actividad familiar u otras situaciones personales que influyen en la dedicación y que anteriormente impedían o dificultaban el acceso a la formación. Con la utilización de las computadoras y las TIC'S, estos nuevos colectivos pasan a formar comunidades de aprendizaje que interactúan y se preparan para colaborar a través del trabajo en equipo. Esta interacción permite igualmente estrechar los vínculos en la comunidad formada por el profesor y el grupo de estudiantes. La comunidad no basa su interacción en el consumo de información, sino que tiene capacidad para producirla y distribuirla.

En este contexto los estudiantes, cuya principal tarea consiste en la creación de conocimiento utilizando como fuente la intervención del profesor sobre la información, y al amplio acceso a bancos de recursos, información y contenidos, se convierten en elaboradores de contenido. Éstos ven aumentada su autonomía, regulada a través de las estrategias de aprendizaje basadas en el objetivo de aprender a aprender (Agora).

Muchas veces el estudiante toma esto como una obligación que cumplir, y que debe obtenerla y nada más, no importa en donde haga la práctica, lo que importa es cumplir el requisito previo, sin embargo la misma juventud a veces bloquea las aspiraciones futuras, no analiza que en un futuro muy cercano se enfrentara a una realidad social, que es "la búsqueda de un empleo en el mercado laboral"

Situación actual de las prácticas pre profesionales en la Universidad de Guayaquil

Ahora bien, las practicas pre profesionales, sería esa experiencia que el estudiante necesitaría para poder enfrentar esas oportunidades que se encuentran en el "mercado laboral", sin embargo existen diversos resultados de la aplicación de esta herramienta de formación académica.

Los resultados esperados de aquel primer acercamiento a un ambiente laboral real, no es tan satisfactorio como pretende el estudiante, muchas veces la falta de control y seguimiento por parte del tutor académico hace que el desarrollo de esa práctica pre profesional no se ejecute según lo esperado para esta actividad.

En variadas ocasiones los empresarios no dan oportunidad al desarrollo académico del estudiante, algunas veces por desconfianza de que el estudiante no desarrolle como se espera, otras veces porque piensan que el estudiante no tiene la madurez necesaria para enfrentar una problemática o porque como no forma parte de la empresa, las actividades encomendadas no las ejerce con la responsabilidad del caso.

Debido a estas posturas, las empresas optan por enviar al estudiante a realizar actividades para las cuales no fue destinado el estudiante en la práctica, tales como archivar, sacar copias, 
numerar documentos, entregar turnos a los clientes, y diversas actividades que no forman parte de su formación académica.

En otros casos, la falta de compromiso del estudiante tiene como resultado en diversas ocasiones que la finalidad de la práctica pre profesional no sea acorde a lo esperado, esta falta de compromiso muchas veces se da porque el estudiante no está de acuerdo en hacer la práctica por diversas aristas tales como, indiferencia a sus estudios, no se siente capaz de actuar en una organización, temor a trabajar en equipo en una empresa, pero como es obligatorio en el régimen académico pues no le queda de otra que hacer la práctica, y una vez mas no se logra lo esperado.

Por ultimo ese seguimiento por parte del tutor académico, en el cual controle las actividades del estudiante para ejerza ese compromiso el cual sostuvo realizar al momento de empezar la práctica, y en las empresas para que cumpla aquel compromiso que describió al momento de aceptar al estudiante, es bastante escueto por lo que se tiene como resultado la pérdida del resultado esperado en las practicas pre profesionales.

Marco Legal que regula las prácticas pre profesionales en educación superior.

Si revisamos la ley orgánica de educación superior LOES (2010), en su artículo 87, menciona que uno de los requisitos previos a la obtención del título de tercer nivel, es que los estudiantes deben acreditar servicios a la comunidad mediante prácticas o pasantías pre profesionales.

Si bien es cierto lo que menciona la LOES en relación a los requisitos para ser profesional en el Ecuador es clara, no hay duda que la intención de esta ley es que el estudiante participe de manera activa con la sociedad, dando a conocer sus fortalezas adquiridas en el plano académico, sin embargo no menciona en que momento de su formación académica debería ejecutar la practica el estudiante, se puede entonces determinar que esta acción se la deja a otra función del estado como lo es el CES, que se encarga de velar por las actividades de educación superior.

Según el CES (2013), en el capítulo 3 artículo 89 indica lo siguiente; Son actividades de aprendizaje orientadas a la Aplicación de conocimientos y al desarrollo de destrezas y habilidades específicas que un estudiante debe adquirir para un adecuado desempeño en su futura profesión. Estas prácticas deberán ser de investigación-acción y se realizarán en el entorno institucional, empresarial o comunitario, público o privado, adecuado para el fortalecimiento del aprendizaje. Las prácticas pre profesionales o pasantías son parte fundamental del currículo conforme se regula en el presente Reglamento.

El artículo en su inicio habla de aplicación de conocimientos, destrezas, habilidades específicas, si esto menciona el reglamento, pues se debe entender que estas aptitudes solo se podrían desarrollar en un nivel superior, ya que los primeros niveles de formación son de instrucción básica, aptitudes que no están adquiridas aun por los estudiantes en su educación básica - profesional, sino más bien estarían en capacidad en un nivel de formación profesional titular, quiere decir en los últimos niveles de su preparación académica, sin embargo el artículo no lo específica y las IES ejecutan su desarrollo en diversos niveles académicos. 
En el capítulo 3 artículo 94 párrafo 1 indica lo siguiente; Las actividades de servicio a la comunidad contempladas en los artículos 87 y 88 de la LOES serán consideradas como prácticas pre profesionales. Para el efecto. Se organizarán programas y proyectos académicos que deberán ejecutarse en sectores urbano-marginales y rurales. Estas prácticas tendrán una duración mínima de 160 horas del mínimo de 400 horas de prácticas pre profesionales establecidas en el artículo 89 del presente Reglamento, inclusive para la modalidad dual.

Es de suma importancia estas cita que se enuncia en el Reglamento de régimen académico establecido por el CES, debido a que determinan la actividad de la práctica del estudiante de las IES en un espacio de tiempo, se puede inferir según lo que menciona la reglamentación que las prácticas laborales son 240 horas de las 400 que exige como cumplimiento de todos los estudiantes de las IES.

Sin embargo no especifica el espacio de tiempo, si las debe realizar en forma continua o podría hacerlas parcialmente, por lo que las IES, pueden parcializar las horas, diversas cantidad de horas en varios niveles, lesionando la espiritualidad y la finalidad de la práctica pre profesional, si lo que se busca realmente es el desarrollo de esas aptitudes del estudiante como menciona el artículo 89 de este reglamento, mal se haría en parcializar las horas en diferentes niveles.

El escenario ideal sería que el estudiante tendría que desarrollar sus actividades de prácticas pre profesionales en un tiempo de 2 meses, tomando en cuenta que máximo se ejecuten 6 horas diarias, debido a que si ejecuta 8 horas diarias se transformaría en una jornada laboral.

Según el Código de Trabajo (2012) indica en el Art. 47.- De la jornada máxima.- La jornada máxima de trabajo será de ocho horas diarias, de manera que no exceda de cuarenta horas semanales, salvo disposición de la ley en contrario, lo que ocasionaría una extinción de la práctica, también podrían ser menos horas, y obviamente el tiempo de ejecución de la práctica pre-profesional se extendería un poco más.

En el mismo inciso en el párrafo 4 indica lo siguiente; Para el desarrollo de las prácticas pre profesionales, cada IES establecerá convenios o cartas de compromiso con las contrapartes públicas o privadas. Como parte de la ejecución de los mismos deberá diseñarse y desarrollarse un plan de actividades académicas del estudiante en la institución receptora.

En relación a este párrafo del artículo 94, el CES busca establecer un compromiso legal entre la IES y la contraparte, lo que en efecto le da la formalidad y seriedad del caso, sin embrago esta figura de convenios limita un poco la ejecución de la práctica del estudiante, debido a que gran cantidad de empresarios no están dispuestos a firmar convenios con las IES, por diversas situaciones, en unos casos el empresario no es de la ciudad, es extranjero, no nota ninguna ventaja en este tipo de compromisos, en fin casos diferentes y circunstancias que como resultado dan la no firma de estos convenios, y como consecuencia a esto el estudiante no tiene donde ejecutar sus prácticas.

Las prácticas pre profesionales como fuente de nuevos aprendizajes y desarrollo de competencias 
Ante esta situación, cabe reflexionar, una definición propia que deberían hacerse las IES, desglosar la frase; "practicas pre profesionales", si se analiza cada palabra buscando el significado en el RAE, la primera definición de "prácticas" se enuncia de la siguiente manera; ejercitar, poner en práctica algo que se ha aprendido o especulado otra definición dice; realizar las prácticas que permiten a alguien habilitarse y poder ejercer públicamente su profesión.

Como sabemos el pre es un prefijo según la (Real Acadmeia de la Lengua) estos son elementos afijos, carentes de autonomía, que se anteponen a una base léxica (una palabra o una expresión pluriverbal) a la que aportan diversos valores semánticos, que implica antes de. Consecuentemente la última palabra de esta frase seria "Profesional", la cual nos dice lo siguiente; Perteneciente o relativo a la profesión, otra definición nos dice que ejerce su profesión con capacidad y aplicación relevantes.

Si verificamos la definición de cada palabra nos damos cuenta que en una de ellas (practicas) para ser exacto, nos menciona poner en práctica lo aprendido, en efecto en los primeros niveles aún no se ha aprendido más que la información de desarrollo general, de la formación académica del estudiante, mas no ha aprendido lo especifico de su profesión.

La otra palabra profesional, se dice que es cuando ejerce su profesión con capacidad y aspectos relevantes, sencillo de entender, un estudiante no puede ser profesional en los primeros niveles de formación, ya debe estar concluyendo sus estudios en la IES.

Entonces para lograr el espíritu de la práctica pre profesional que según hemos revisado es la primera experiencia laboral real que el estudiante de la IES desea tener dentro de su perfil de formación, avalado, concientizado, vivenciado, se debe tomar en cuenta un análisis profundo por parte de las IES, ante la verdadera necesidad del estudiante en esta actividad académica.

Tomando en cuenta esa conceptualización de la frase "practicas pre profesionales", que realmente llene esa expectativa que tiene el estudiante de esta actividad, que no sean casos aislados la inserción laboral mediante la práctica del estudiante en las empresas, más bien que sea una gran posibilidad y que realmente cumpla con ese primer objetivo de esta actividad que sería la primera experiencia laboral.

La vinculación de la teoría y de la práctica en las prácticas pre profesionales

Matos \& Pérez (2012) manifiestan que los avances de la ciencia, la tecnología y el desarrollo social, como procesos que están estrechamente relacionados, imponen a las diferentes instituciones la formación de un estudiante capaz de insertarse en la práctica con una preparación que le permita estar al alcance de dichos procesos. Estos autores corroboran que cada día cobra mayor fuerza la necesidad de formar un estudiante capaz de movilizar su actividad a la vida social, a lo laboral, atendiendo a sus raíces, tradición e historia, para poner en práctica los conocimientos aprendidos durante su formación.

Díaz (2005) señala que estas tendencias de enseñanza dependen de su fin; el estudio de casos tiene como finalidad la adquisición del aprendizajes mediante el análisis de casos reales o simulados, en consecuencia, la relación teoría y práctica como forma de enseñanza se da en un entorno que relaciona la universidad y la empresa en un mismo contexto real, estudio de casos 
como método es un análisis intensivo y completo de un hecho, problema o suceso real, con la finalidad de conocerlo, interpretarlo, resolverlo, generar hipótesis, contrastar datos, reflexionar, completar conocimientos, diagnosticar y en ocasiones, entrenarse en los posibles procedimientos alternativos de solución.

Los fundamentos de la teoría pedagógica tienen dos salidas: procesos cognitivos y procesos afectivos, el primero permite determinar una adecuación al objeto con ideas, reflexiones y se analiza el comportamiento. En los procesos afectivos la imagen de relación con el objeto, mediante las necesidades se manifiesta con depresión, ansiedad, pesimismo, sentimientos, estado de ánimo. Su comportamiento puede darse de manera física y psíquica, dan una especificidad de la personalidad que el estudiante adopta generando un control y regulación de la conducta, es decir, su personalidad, tan importante para lograr la eficacia de los resultados del aprendizaje y su impacto en la gestión de la función de gestión de conocimientos, como resultado de su formación (Macas \& León, 2016).

Los ambientes de formación, en especial los de trabajo como práctica, influyen directamente en el comportamiento de la personalidad del futuro profesional, las influencias personales y sociales pueden generar o contribuir positiva o negativamente en la personalidad del individuo, de ahí el cuidado con las influencias, en especial las de trabajo que puedan ejercer sobre sí mismo; la educación en valores como construcción individual es de vital importancia para todo tipo de formación profesional (Macas \& León, 2016).

Frente a esta situación del estudiante, cobra mayor importancia el vínculo de movilizar cada vez más hacia el aspecto social, en este caso al trabajo, pero con una preparación que garantice la eficacia y eficiencia de su desempeño laboral como administrador, las acciones comprometen la supervivencia económica y social de la empresa productiva, que propicie el desarrollo de la sociedad y del ser como persona y su entorno familiar.

Frente a las situaciones expuestas y en relación al análisis de la frase, se puede establecer un debate en el cual permita la concientización de la ejecución de esta actividad académica, partiendo como primer punto la negatividad a realizar prácticas en la misma IES, debido a que en el $90 \%$ de las carreras no realizarían las vivencias laborales reales, en segunda instancia se debería establecer que la ejecución de estas prácticas deben ser en el último nivel de formación académica.

Principales dificultades reportadas por los estudiantes en el desarrollo de las prácticas pre profesionales en la Universidad de Guayaquil.

Existen casos diversos de empresas en que la oportunidad de desarrollo no les fue otorgada a los estudiantes, muchas veces por el sigilo de la información, desconfianza en el desarrollo del estudiante, temor de dar cabida a un posible candidato, varios factores que llevaron a que el estudiante no desarrolle esa experiencia que se busca en realidad obtener de esta actividad académica, como son las practicas pre profesionales.

Otros casos en que los empresarios se valieron de esta herramienta educativa para llenar esos puestos de trabajo, para los cuales no quieren pagar a un empleado y trataron de usar a los 
estudiantes, como repartidores de volantes, como mensajería, entorpeciendo el desarrollo académico pare le cual se comprometió la empresa.

En diversas ocasiones el desconocimiento de ciertas actividades llevo consigo al desinterés por parte del empresario en el estudiante, puesto que espero mucho más conocimiento del practicante al momento de encomendarle diversas actividades, en virtud de esa respuesta lo envió a realizar actividades sin importancia, lo cual llevo al practicante a desertar de la práctica por vergüenza ante el desconocimiento de las tareas encomendadas.

Es importante entonces enfocar esa finalidad de la práctica pre profesional, de tal manera que cuando el estudiante salga a desarrollar lo aprendido, este acorde con lo esperado por el empresario, siendo esta sinergia entre estos actores lo esperado por esta herramienta de formación académica.

\section{Propuesta de mejora de las prácticas pre profesionales en la Universidad de Guayaquil}

Entonces, ¿Que nos quiere decir que desarrollemos el CES en el estudiante?, mucho dependería de que se quiera lograr como IES en sus alumnos, se debe entender que la gran mayoría de carreras debe ejercer la práctica pre profesional, fuera de la IES, debe ser en el mercado laboral, donde realmente existe las vivencias acorde a su economía, la academia busca dar a conocer una guía general de cómo enfrentar diversos acontecimientos, sin embargo al momento de formar parte de la realidad laboral, existen situaciones específicas, ya no generales como se aprendió en clases, y esas soluciones nos la ofrece la experiencia laboral.

Por otro lado el seguimiento a la práctica por uno de los actores como lo es el tutor académico es de suma importancia, de este actor depende mucho el hacer cumplir las obligaciones y responsabilidades tanto del empresario como el estudiante, buscando de esta manera obtener con efectividad la finalidad de las practicas pre profesionales, que como se ha expuesto en este escrito, que sería esa primera experiencia laboral real mediante esta herramienta educativa.

Sin embargo para ese seguimiento por parte del tutor académico se debe considerar, las diversas actividades administrativas que como docentes tienen estos tutores, buscando dejar en ellos únicamente esta actividad de tal manera que puedan lograr ejercer ese control necesario que dé como resultado esa finalidad esperada que busca la práctica pre profesional.

Como se puede revisar en este escrito el reglamento de régimen académico expedido por el CES, limita las oportunidades de ejecutar las practicas pre profesionales, al exponer esta modalidad de convenios con entidades jurídicas, se debe buscar la flexibilidad de desarrollo de las prácticas pre profesionales en pequeños negocios, en la actualidad las personas naturales obligadas a llevar contabilidad no son tomadas en cuenta para que los estudiantes puedan ejercer la práctica pre profesional, mermando de esta manera las oportunidades de resolver esta actividad estudiantil.

Concientizar en el estudiante el verdadero espíritu académico de las prácticas pre profesionales exponiendo las bondades que darán para su futura inclusión en el mercado laboral, logrando el compromiso de ellos al momento de ejercer su actividad. 


\section{Bibliografía}

Agora. (s.f.). papel del alumno. Obtenido de http://agora.ucv.cl/manual/rol_alumno\%5Crol_alumno.html

CES. (12 de 11 de 2013). Consejo de Educación Superior. Obtenido de http://www.ces.gob.ec/doc/Reglamentos_Expedidos_CES/codificacin\%20del\%20reglam ento\%20de\%20rgimen\%20acadmico.pdf

Collinson, V., Kozina, E., Lin, Y.-H. K., Ling, L., Matheson, I., Newcombe, L., y otros. (2009). Professional development for teachers: A world of change. European.

Derrick, J., \& Dicks, J. (2005). Teaching Practice and Mentoring: The key to effective literacy, language and numeracy teacher training. Leicester: National Institute of Adult Continuing Education.

Díaz, M. (2005). Modalidades de enseñanza centradas en el desarrollo de competencias. Orientadas para promover el cambio metodológico en el espacio europeo de educación superior.

DiLorenzo-Aiss, J. a. (1996). Marketing higher education:Models of marketing internship programs as tools for the recruitment and retention of marketing internship programs as tools for the recruitment and retention of undergraduate majors. Journal of Marketing for Higher Education 7 .

Ferreyra, M. G. (2007). Determinantes del desempeño universitario: efectos heterogéneos en un modelo censurado. . Argentina: (Tesis de Maestría en Economía, Universidad Nacional de La Plata, Argentina.

Gault, J. R. (2000). Undergraduate business internships and career success: are they related? Journal of marketing education.

LOES. (12 de 10 de 2010). Ley Organica de Educación Superior. Recuperado el 30 de 12 de 2016, de https://procuraduria.utpl.edu.ec/sitios/documentos/NormativasPublicas/Ley\%20Org\%C3 $\%$ A1nica\%20de\%20Educaci\%C3\%B3n\%20Superior\%20Codificada.pdf

Macas, H. F., \& León, M. I. (2016). Análisis de la integración de teoría y la práctica de la disciplina de administración de operaciones en la formación de administradores de empresas, reflexión desde lo académico y laboral. Mi SciELO.

Matos, E. \&. (2012). Qué entender por formación laboral con perfil identitario.

Real Acadmeia de la Lengua. (s.f.). Obtenido de http://www.rae.es/consultas/normas-de-escriturade-los-prefijos-exmarido-ex-primer-ministro 
Rodríguez, J., \& González, L. (1995). Recursos humanos, educación y desarrollo en la perspectiva Latinoamericana y del Caribe. Santiago-Chile: CEPAL/ CELADE.

Trabajo, C. d. (26 de 09 de 2012). Código de Trabajo. Obtenido de http://www.trabajo.gob.ec/wpcontent/uploads/downloads/2012/11/C\%C3\%B3digo-de-Tabajo-PDF.pdf

Yáñez, C. E., Soto, Y. M., Domínguez, N. A., \& Hacegaba, G. Y. (19 de 09 de 2014). Aprender a hacer:la importanciade las prácticas profesionales docentes. Redalyc, 9.

Zabalza Beraza, M. (Enero-Abril de 2006). El Practicum y la formación del profesorado:balance y propuesta para las nuevas titulaciones. En J. M. Escudero, La mejora de la educación y la formación del profesorado. Políticas y prácticas (págs. 309-330). Barcelona: Octaedro. 